\title{
Short Term Alterations of Hormone Profile Following Oocyte Pick-Up in Women with Polycystic Ovarian Syndrome to Assess the Effect of Multiple Needle Entries Into the Ovarian Cortex and Stroma
}

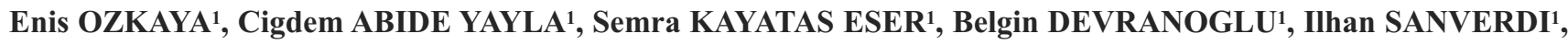 \\ Tayfun KUTLU1 \\ Istanbul, Turkey
}

ABSTRACT

OBJECTIVE: Some hormonal alterations after ovarian diathermy have been proposed in previous studies. Based on this data, we aimed to analyze some hormone profile changes following oocyte pick-up in women with polycystic ovarian syndrome.

STUDY DESIGNS: A total of 50 women with polycystic ovarian syndrome underwent ovarian stimulation for IVF cycle with an indication of anovulatory infertility. Some hormone profiles with the insulin resistance were assessed before and after oocyte pick-up to (early in the morning before starting stimulation and repeated 1 month later from oocyte pick-up day) assess whether high number of needle entries into the ovarian cortex and stroma result in similar effect with the diathermy.

RESULTS: Comparison of some variables revealed significantly decreased HOMA-IR and serum total testosterone concentrations after intervention. Correlation analyses showed significant correlations between number of needle entries, change in HOMA-IR, baseline total testosterone level and change in AMH level.

CONCLUSION: Our data showed significant metabolic and hormonal alterations following oocyte pick up consistent with the effect of ovarian diathermy in women with polycystic ovarian syndrome.

Keywords: Ovarian puncture, Polycystic ovarian syndrome, Diathermy, Testosterone, Insulin resistance Gynecol Obstet Reprod Med 2018;24(1):17-21

\section{Introduction}

Ovarian diathermy was introduced as a treatment option for anovulatory patients with polycystic ovarian syndrome (PCOS) $(1,2)$. While there is no data regarding the exact mechanism of this approach, it is well known that ovarian damage by any method result in similar endocrinological alterations (3). Several articles have been published about the

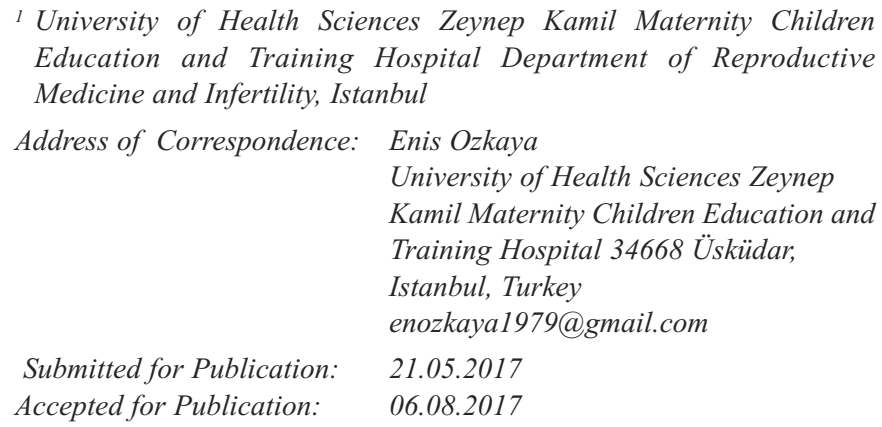

\begin{tabular}{|c|c|}
\hline \multicolumn{2}{|c|}{ Access this article online } \\
\hline 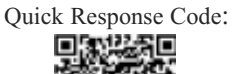 & $\begin{array}{l}\text { Website: www.gorm.com.tr } \\
\text { e- mail: info@gorm.com.tr }\end{array}$ \\
\hline 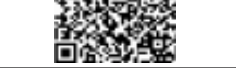 & DOI: 10.201613/GORM.2017.713 \\
\hline
\end{tabular}

How to cite this article: Ozkaya E. Abide Yayla C. Kayatas Eser S. Devranoglu B. Sanverdi I. Kutlu T. Short Term Alterations of Hormone Profile Following Oocyte Pick-Up in Women with Polycystic Ovarian Syndrome to Assess the Effect of Multiple Needle Entries Into the Ovarian Cortex and Stroma. Gynecol Obstet Reprod Med 2018;24(1):17-21 endocrine consequences of these procedures. Some theories have been proposed for the exact mechanism of this procedure including reduced size of ovary to allow gonadotrophins to act more effectively and increased blood flow to the ovaries (4-6).

Whatever the exact mechanism, studies are consistent about some endocrine alterations secondary to these procedures such as less (free) androgens, lower luteinizing hormone (LH) levels and lower anti-Mullerian hormone (AMH) production $(7,8)$. And secondary to these endocrine alterations, an increased ovarian responsiveness to FSH has been proposed (7).

There is also an argument that the mechanical manipulation/movement of the ovaries itself may be the main cause of this effect rather than the diathermy. Therefore, a recent study showed significantly decreased LH concentrations just after mechanical movement of the ovaries by an ultrasound probe in PCOS patients, a neuronal pathway from the ovaries communicating to the hypothalamic-pituitary system was proposed to be the most reasonable explanation (9).

Based on this data, we aimed to analyze some hormone profile changes following oocyte pick-up that result in both mechanical movement and injury of the ovary in women with PCOS.

\section{Material and Method}

This study has been approved by the ethics committee of 
Zeynep Kamil Women and Children's Health Training and Research Hospital, Istanbul, Turkey. An informed consent was obtained from each participant. This prospective, single-center cohort study was performed on the infertile women who underwent assisted reproductive technologies treatment in IVF clinic of Zeynep Kamil Women and Children's Health Training and Research Hospital, Istanbul, Turkey between July 2016 to November 2016. Seventy-four women recruited for baseline metabolic and hormone analyses, fifty cases without embryo implantation were included in the final analyses. All participants underwent ICSI cycles with antagonist protocol in the ART center.

Demographic features and medical information regarding menstrual cycles, obstetric and gynecological history, and previous medication and/or disease were collected from the medical records. Body mass index (BMI) was calculated as body weight (in kilograms) divided by height (in meters squared). The waist to hip ratio $(\mathrm{W} / \mathrm{H})$ was calculated from dividing the waist circumference by the hip circumference. PCOS was diagnosed according to the Rotterdam criteria with the existence of at least two of the following three features: 1) oligo- or amenorrhea; 2) clinical or biochemical hyperandrogenism; or 3) polycystic ovarian morphology on pelvic ultrasound, after exclusion of other causes of hyperandrogenism, thyroid disorders, hyperprolactinemia.

Inclusion criteria were normal serum prolactin levels and without hormone treatment (oral contraceptives, progestin etc.) within 3 months. The patient ages ranged from 20 to 39 years. In all patients assisted reproductive techniques were indicated for anovulatory infertility.

Exclusion criteria were previous ovarian surgery, oral contraceptive or metformin use within the last 3 months, mechanical or male subfertility and co-existing endocrine diseases (diabetes mellitus, estrogen-dependent tumors, thyroid disease, Cushing's syndrome or congenital adrenal hyperplasia).
Antagonist (Cetrorelix, Merck-Serono, Geneva, Switzerland) $0.25 \mathrm{mg}$ /day was administered when the follicular size was 12 $\mathrm{mm}$. After the follicular size reached $18 \mathrm{~mm}$, rhCG $250 \mu \mathrm{g}$ was administered, and follicular puncture was performed after 34-36 hours by 17-gauge double-lumen needle (Cook Ireland Ltd., Limerick, Ireland). Endocrine measurements were performed early in the morning before starting stimulation and repeated 1 month later from oocyte pick-up day in women who did not conceive in the first attempt.

\section{Endocrine measurements}

Serum total testosterone levels were measured by immunoassay method (Advia Centaur XP, Siemens, Erlangen, Germany). Glucose and insulin levels were measured using the enzyme- linked immunosorbent assay (ELISA) method. IR was calculated by homeostasis model of assessment (HOMA) according to fasting blood glucose and insulin levels $[$ HOMA-IR $=$ fasting glucose level $(\mathrm{mg} / \mathrm{dL}) \times$ fasting insulin level $(\mathrm{mIU} / \mathrm{mL}) / 405]$. Change in $\mathrm{AMH}$, testosterone and HOMA-IR levels were calculated by formula: the level before OPU-the level after OPU

\section{Primary outcome}

Some metabolic and endocrine alterations secondary to oocyte pick up.

\section{Statistical analysis}

Data were analyzed using SPSS 15.0 for Windows. Paired samples $t$ test was used to compare continuous variables within the groups. Wilcoxon signed rank test was used to compare non-normally distributed continuous variables within the groups before and after intervention. Correlation analyses were used to show correlations between variables. $p$ value $<$ 0.05 was accepted to be statistically significant.

\section{Results}

Some clinical and demographic characteristics of whole study population were summarized in table 1 .
Follicle monitorization was performed by two dimensional sonographic measurements of growing follicles and calculating the mean value at each visit. Antagonist protocol was used in all cases; on the second day of the menstrual cycle, ampules of rFSH (Gonal-F, Merck-Serono, Geneva, Switzerland) 75-150 IU, depending on patient's response, were administered and follicular growth was monitored using transvaginal sonography. The dosage of rFSH was adjusted from day 5 of stimulation according to the ovarian response.
Table 1: Summary of some clinical and demographic characteristics of whole study population

\begin{tabular}{lrrrr}
\hline & Mean & Std. Deviation & Minimum & Maximum \\
\hline Age (Years) & 28.5 & 3.6 & 23 & 36 \\
Duration of infertility (Years) & 5.8 & 3.3 & 1 & 14 \\
Day 3 FSH (mIU/mL) & 4.9 & 1.3 & 3.30 & 8.18 \\
Day 3 Estradiol (pg/mL) & 41.3 & 14.6 & 25.7 & 76.00 \\
Fasting glucose before OPU (mg/dL) & 95.8 & 26.9 & 68 & 267 \\
Serum insulin before OPU (uU/mL) & 10.9 & 6.9 & 3 & 34.3 \\
Fasting glucose after OPU $(\mathrm{mg} / \mathrm{dL})$ & 89.8 & 7.1 & 72 & 110 \\
Serum insulin after OPU $(\mathrm{uU} / \mathrm{mL})$ & 8.4 & 3.1 & 2 & 15.5 \\
Gonadotropin starting dose $(\mathrm{U})$ & 148.5 & 10.6 & 75.0 & 150 \\
Total gonadotropin dose $(\mathrm{U})$ & 1431.1 & 328.2 & 525.0 & 2400 \\
Duration of stimulation $(\mathrm{Days})$ & 9.6 & 2.03 & 7 & 16 \\
Estradiol at trigger day $(\mathrm{pg} / \mathrm{mL})$ & 2033.9 & 907.1 & 558 & 4117.0 \\
Number of needle entries & 18.8 & 9.4 & 8 & 45 \\
Total oocyte number & 9.6 & 3.8 & 6 & 24 \\
\hline
\end{tabular}


Comparison of some variables before and after oocyte pick-up was shown in table 2 indicating significantly decreased HOMA-IR and serum total testosterone concentrations after intervention. Correlation analyses revealed significant correlations between the number of needle entries, change in HOMA-IR, total testosterone concentration, baseline total testosterone level and the change in $\mathrm{AMH}$ level (Table 3).

\section{Discussion}

In our study, we aimed to assess some endocrine changes after oocyte pick- up procedure in women with PCOS. Our hypothesis was based on the endocrine changes after ovarian diathermy and the data coming from the study by Hendriks et al., which proposed the mechanical movement/manipulation of the ovaries to be the main confounder for the ovarian response to diathermy procedure (9). Morphological changes in the ovary was claimed to be the underlying mechanism of responses to ovarian drilling (10). During oocyte pick up procedure, both follicular destruction, stromal injury and mechanical manipulation of ovary occur with the needle entry, therefore it is expected to see similar responses to this procedure. In a previous randomized study, ultrasound-guided transvaginal ovarian needle drilling was shown to have similar effect on endocrine profile in women with PCOS, no difference was observed between groups with ovarian diathermy and ovarian needle drilling in terms of rate of regular menses, hirsutism, acne, regular ovulation, pregnancy, serum $\mathrm{LH}$, $\mathrm{FSH}, \mathrm{FSH} / \mathrm{LH}$ ratio and testosterone levels (11). Spontaneous ovulation is the main goal for this intervention, therefore some endocrine changes in the $\mathrm{AMH}$, testosterone, free androgen index, and LH were studied and shown to be associated with ovulatory response after unilateral or bilateral diathermy in women with polycystic ovary syndrome (12). In their study, authors showed the value of testosterone achieved in the 1st month after the diathermy as one of the strongest and consistent determinant of ovulation response. Based on this data, we assessed hormone profiles at 1st month after oocyte pick up procedure and our data showed significantly decreased testosterone levels. According to the pathophysiological point of view, a disorder in folliculogenesis was shown to be associated with increased production of androgens and AMH by the antral follicles in women with PCOS. And diathermy was proposed to decrease in these antral follicles which lead to folliculogenesis and ovulation (13). In our data, there were decreased AMH levels after procedure but the difference did not reach statistical significance. And degree of decrement in AMH levels were significantly correlated with number of needle entries and degree of decrement in serum testosterone levels. Although there is no consensus on this issue, another study pointed the possible beneficial effect of androgen-producing stroma destruction as the critical effect of procedure (14). In majority of the cases with multiple follicle development, needle is needed to be inserted all through the stroma in order reach the follicles located at the upper site of the ovary. These invasive interventions were thought to result in similar effect with the diathermy that lead to metabolic alterations.

Some variables were introduced that affect the treatment success of diathermy including the amount of thermal energy (15), number of punctures, power used, and duration of each punctures (16). Higher number of punctures with higher doses of thermal energy resulted in the reductions of free androgen index and testosterone (17). It seems that higher degree of tissue destruction result in better results in this procedure. Generally, it is expected to see higher amount of destruction in cases with multiple follicle development by multiple needle entries.

Table 2: Comparison of some variables before and after oocyte pick-up indicating significantly decreased HOMA-IR and serum total testosterone concentrations after intervention

\begin{tabular}{lccl}
\hline & Before & After & $p$ value \\
\hline HOMA-IR & $2.7 \pm 0.6$ & $1.8 \pm 0.7$ & $<0.05$ \\
$\mathrm{~T}(\mathrm{nmol} / \mathrm{L})$ & $1.04 \pm 1.3$ & $0.4 \pm 0.013$ & $<0.05$ (Wilcoxon signed rank test) \\
$\mathrm{W} / \mathrm{H}$ ratio & $0.85 \pm 0.07$ & $0.86 \pm 0.08$ & NS \\
$\mathrm{AMH}(\mathrm{pg} / \mathrm{dL})$ & $7.35 \pm 4.9$ & $5.8 \pm 3.1$ & NS (Wilcoxon signed rank test) \\
$\mathrm{BMI}\left(\mathrm{kg} / \mathrm{m}^{2}\right)$ & $26.6 \pm 4.4$ & $26.9 \pm 4.5$ & NS \\
\hline
\end{tabular}

T: Testosterone, W/H: Waist/hip ratio, BMI: Body mass index

Table 3: Correlation analyses revealed significant correlations between number of needle entries, change in HOMA-IR, total testosterone concentration, baseline total testosterone level and change in $\mathrm{AMH}$ level

\begin{tabular}{rrrrrr}
\hline & & No of needle entries & Change in HOMA-IR & Change in T & Baseline T \\
\hline & $\mathrm{r}$ & 0.502 & -0.109 & 0.742 & 0.744 \\
Change in AMH & $\mathrm{p}$ & 0.009 & 0.589 & 0.000 & 0.000 \\
& $\mathrm{n}$ & 50 & 50 & 50 & 50 \\
\hline
\end{tabular}

T: Testosterone, $r$ : correlation coefficient, $p: p$ value 
Laparoscopic ovarian drilling was thought to result in favorable intra-ovarian and systemic endocrinal milieu. Some systemic endocrinal changes were shown such as decreased plasma LH and in its pulsations, increased FSH, decreased LH: FSH ratio, a temporary fall in inhibin $\mathrm{B}$, increased sex hormone binding globulin and a constant fall in androgens, free androgen index and Ferriman-Gallwey score $(18,19)$.

Drilling results in the decrease of AMH level that lead to the consideration of higher rate of ovulation in women with greater decline in AMH concentrations (20). We see an insignificant decline in AMH levels after the procedure.

Insulin resistance (IR) is frequently seen in women with polycystic ovarian syndrome and was shown to play a significant role in the pathogenesis of this syndrome (21). Hyperinsulinemia causes disordered folliculogenesis and steroidogenesis that result in oligo/anovulation. BMI and free androgen index were some other confounders for successful outcome after laparoscopic ovarian drilling (22). A multivariate regression model including these three parameters to determine which of the three closely related factors (HOMA index, BMI and free androgen index) is the independent predictor of ovulation after laparoscopic ovarian drilling showed HOMA-IR index to be the most important confounder for the ovulation after laparoscopic ovarian drilling (23). In addition to the decreased total testosterone levels, our data revealed significantly decreased HOMA-IR levels after oocyte pick-up procedure.

Consistent with our result, ovarian electrocautery was shown to result in amelioration of insulin resistance in PCOS (24), however some studies have demonstrated no impact of laparoscopic ovarian drilling on metabolic parameters (25, 26). Additionally, insulin sensitivity and serum glucose levels and lipoprotein profiles remained unchanged in some studies (27). All these studies were conducted on very small number of women so it is expected not to observe significant difference of insulin resistance before and after intervention.

In addition to the tissue destruction by mechanical needle insertion, pathophysiological mechanisms may also include the consequences of inflammatory responses to multiple needle entries supported by the previous data showed deleterious effect of chronic inflammation on the ovarian reserve (28). There are some studies published in the literature that showed elevated serum testosterone levels and insulin resistance following ovarian stimulation $(29,30)$. Therefore, endocrine alterations shown in our study may not be linked to supraphysiologic endocrine milieu secondary to ovarian hyperstimulation.

This is the first study in the literature which showed the effect of needle drilling in PCOS patient underwent ovarian hyperstimulation, although, we do not have the data regarding spontaneous ovulation, both decreased HOMA-IR level and testosterone concentrations in our study, as significant predic- tors, indicated possibility for following ovulatory cycles and better responses in next cycles.

Our data showed significant metabolic and hormonal alterations following oocyte pick up consistent with the effect of ovarian diathermy in women with PCOS. A spontaneous pregnancies or favorable ART outcomes may be expected in selected cases; further investigations are needed to clarify this issue.

: The authors reported no conflict of interest

\section{References}

1. Stein I, Leventhal M. Amenorrhea associated with bilateral polycystic ovaries Am J Obstet Gynecol 1935;29(2): 181-191.

2. Gjonnaess H. Polycystic ovarian syndrome treated by ovarian electrocautery through the laparoscope. Fertil Steril 1984;41(1):20-5.

3. Cohen J. Laparoscopic procedures for treatment of infertility related to polycystic ovarian syndrome. Hum Reprod Update 1996;2(4):337-44.

4. Katz M, Carr PJ, Cohen BM, Millar RP. Hormonal effects of wedge resection of polycystic ovaries. Obstet Gynecol $1978 ; 51(4): 437-44$

5. Ben Shlomo I, Homburg R, Shalev E. Hyperandrogenic anovulation (the polycystic ovary syndrome)-back to the ovary? Hum Reprod Update 1998;4(3):296-300.

6. Takeuchi S, Futamura N, Takubo S, Noda N, Minoura H, Toyoda N. Polycystic ovary syndrome treated with laparoscopic ovarian drilling with a harmonic scalpel. A Prospective, Randomized Study. J Reprod Med 2002;47 (10):816-20.

7. Hendriks ML, Ket JC, Hompes PG, Homburg R, Lambalk $\mathrm{CB}$. Why does ovarian surgery in PCOS help? Insight into the endocrine implications of ovarian surgery for ovulation induction in polycystic ovary syndrome. Hum Reprod Update 2007;13(3):249-64.

8. Elmashad AI. Impact of laparoscopic ovarian drilling on anti-Mullerian hormone levels and ovarian stromal blood flow using three-dimensional power Doppler in women with anovulatory polycystic ovary syndrome. Fertil Steril 2011;95(7):2342-46.

9. Hendriks ML, Konig T, Soleman RS, Korsen T, Schats R, Hompes PG et al. Influence of ovarian manipulation on reproductive endocrinology in polycystic ovarian syndrome and regularly cycling women. Eur J Endocrinol 2013; 169(4):503-10.

10. Hendriks ML, van der Valk P, Lambalk CB, Broeckaert MA, Homburg R, Hompes PG. Extensive tissue damage of bovine ovaries after bipolar ovarian drilling compared to monopolar electrocoagulation or carbon dioxide laser. Fertil Steril 2010;93(3):969-75. 
11. Badawy A, Khiary M, Ragab A, Hassan M, Sherief L. Ultrasound-guided transvaginal ovarian needle drilling (UTND) for treatment of polycystic ovary syndrome: a randomized controlled trial. Fertil Steril 2009 Apr;91 (4):1164-7.

12. Sunj M, Canic T, Jeroncic A, Karelovic D, Tandara M, Juric $\mathrm{S}$ et al. Anti-Müllerian hormone, testosterone and free androgen index following the dose-adjusted unilateral diathermy in women with polycystic ovary syndrome. Eur J Obstet Gynecol Reprod Biol 2014;179:163-9.

13. Fanchin R, Schonauer LM, Righini C, Guibourdenche J, Frydman R, Taieb J. Serum anti-Mullerian hormone is more strongly related to ovarian follicular status than serum inhibin B, estradiol, FSH and LH on day 3. Hum Reprod 2003;18(2):323-7.

14. Gomel V, Yarali H. Surgical treatment of polycystic ovary syndrome associated with infertility. Reprod Biomed Online 2004;9(1):35-42.

15. Amer SA, Li TC, Cooke ID. A prospective dose-finding study of the amount of thermal energy required for laparoscopic ovarian diathermy. Hum Reprod 2003;18(8): 1693-8.

16. Fernandez H, Morin-Surruca M, Torre A, Faivre E, Deffieux X, Gervaise A. Ovarian drilling for surgical treatment of polycystic ovarian syndrome: a comprehensive review. Reprod Biomed Online 2011;22(6):556-68.

17. Weerakiet S, Lertvikool S, Tingthanatikul Y, Wansumrith S, Leelaphiwat S, Jultanmas R. Ovarian reserve in women with polycystic ovary syndrome who underwent laparoscopic ovarian drilling. Gynecol Endocrinol 2007;23 (8):455-60.

18. Kucuk M, Kilic-Okman T. Hormone profiles and clinical outcome after laparoscopic ovarian drilling in women with polycystic ovary syndrome. Med Sci Monit 2005 Jan;11(1):CR29-34.

19. Godinjak Z, Javoric R. Clinical outcome and hormone profiles before and after laparoscopic electroincision of the ovaries in women with polycystic ovary syndrome. Bosn J Basic Med Sci 2007 May;7(2):171-5.

20. Amer SA, Li TC, Ledger WL. The value of measuring anti-Mullerian hormone in women with anovulatory polycystic ovary syndrome undergoing laparoscopic ovarian diathermy. Hum Reprod 2009;24(11):2760-6.

21. Dunaif A, Segal KR, Shelley DR, Green G, Dobrjansky A,
Licholai T. Evidence for distinctive and intrinsic defects in insulin action in polycystic ovary syndrome. Diabetes 1992 Oct;41(10):1257-66.

22. Amer S, Li TC, Ledger WL. Ovulation induction using laparoscopic ovarian drilling in women with polycystic ovarian syndrome: predictors of success. Hum Reprod 2004 Aug;19(8):1719-24.

23. Amer S, Li TC, Samuelson C, Ledger W. The impact of insulin resistance on the outcome of laparoscopic ovarian diathermy in women with polycystic ovarian syndrome. Acta Obstet Gynecol Scand 2011;90(4):338-43.

24. Seow KM, Juan CC, Hsu YP, Hwang JL, Huang LW, Ho LT. Amelioration of insulin resistance in women with PCOS via reduced insulin receptor substrate-1 Ser 312 phosphorylation following laparoscopic ovarian electrocautery. Hum Reprod 2007 Apr;22(4):1003-10.

25. Lemieux S, Lewis GF, Ben-Chetrit A, Steiner G, Greenblatt EM. Correction of hyperandrogenemia by laparoscopic ovarian cautery in women with polycystic ovarian syndrome is not accompanied by improved insulin sensitivity or lipid-lipoprotein levels. J Clin Endocrinol Metab 1999 Nov;84(11):4278-82.

26. Tiitinen A, Tenhunen A, Seppälä M. Ovarian electrocauterization causes LH-regulated but not insulin-regulated endocrine changes. Clin Endocrinol (Oxf). 1993 Aug;39 (2):181-4.

27. Tulandi T, Saleh A, Morris D, Jacobs HS, Payne NN, Tan SL. Effects of laparoscopic ovarian drilling on serum vascular endothelial growth factor and on insulin responses to the oral glucose tolerance test in women with polycystic ovary syndrome. Fertil Steril. 2000 Sep;74(3):585-8.

28. Cui L, Sheng Y, Sun M, Hu J, Qin Y, Chen ZJ. Chronic Pelvic Inflammation Diminished Ovarian Reserve as Indicated by Serum Anti Mülerrian Hormone. PLoS One. 2016;11(6):e0156130. doi:10.1371/journal.pone.0156130.

29. Fanchin R, de Ziegler D, Taieb J, Olivennes F, Castracane VD, Frydman R. Human chorionic gonadotropin administration does not increase plasma androgen levels in patients undergoing controlled ovarian hyperstimulation. Fertil Steril 2000;73(2):275-9.

30. Ma Q, Fan J, Wang J, Yang S, Cong Q, Wang R et al. High levels of chorionic gonadotrophin attenuate insulin sensitivity and promote inflammation in adipocytes. J Mol Endocrinol 2015;54(2):161-70. 